\title{
The effect of psychoeducational intervention, based on a self-regulation model on menstrual distress in adolescents: a protocol of a randomized controlled trial
}

\author{
Somayeh Asgari ${ }^{1}$, Zainab Alimoardi ${ }^{2}$, Mohammad Ali Soleimani ${ }^{2}$, Kelly-Ann Allen ${ }^{3}$ and Nasim Bahrami ${ }^{*}$ (1)
}

\begin{abstract}
Introduction: Menstrual distress caused by primary dysmenorrhea is associated with physical and psychological symptoms - before, after, and during menstruation. Leventhal's self-regulation educational model is based on the cognitive and emotional experiences of threat responses to symptoms and relates to coping responses. This study aims to investigate the effect of the implementation of a psychoeducational intervention, based on the selfregulation model of menstrual distress in adolescents.

Methods/design: In this randomized controlled trial, 120 adolescent girls with moderate to severe menstrual pain (based on visual analog scale (VAS) $\geq 4$ ) from twelve randomly selected high schools in Qazvin City will be enrolled in the study and will be randomly assigned to either a 3-session psychoeducational intervention $(n=60)$ or control $(n=60)$ groups. The sessions will be between 60 and 90 min apiece, and they will run for three consecutive weeks (one session per week). The data collection tools will include questionnaire eliciting menstrual information and demographics, the VAS, the Moos Menstrual Distress Questionnaire, and the illness perception questionnaire. One month prior to the intervention, both groups will participate in an initial assessment to assess the severity of their pain and level of menstrual distress. Finally, all questionnaires will be completed for three consecutive months after the intervention is completed.
\end{abstract}

Discussion: It is anticipated that findings of this study will provide evidence for the effectiveness of the Leventhal self-regulation model. Implications for improved practice, understanding, and treatment for menstrual distress may also arise.

Ethical considerations: The research protocol will be reviewed by the ethics committee, which is affiliated with the Qazvin University of Medical Sciences (Decree code: IR.QUMS.REC.1398.043).

Trial registration: IRCT20190625044002N1. Registration date: 2019-09-03.

Keywords: Menstrual distress, Dysmenorrhea, Illness perceptions, Self-regulation model

\footnotetext{
* Correspondence: nbahrami@qums.ac.ir

${ }^{2}$ Social Determinants of Health Research Center, Research Institute for

Prevention of Non-Communicable Diseases, Qazvin University of Medical

Sciences, Shahid Bahonar Blvd, Qazvin 3419759811, Iran

Full list of author information is available at the end of the article
}

(c) The Author(s). 2020 Open Access This article is licensed under a Creative Commons Attribution 4.0 International License, which permits use, sharing, adaptation, distribution and reproduction in any medium or format, as long as you give appropriate credit to the original author(s) and the source, provide a link to the Creative Commons licence, and indicate if changes were made. The images or other third party material in this article are included in the article's Creative Commons licence, unless indicated otherwise in a credit line to the material. If material is not included in the article's Creative Commons licence and your intended use is not permitted by statutory regulation or exceeds the permitted use, you will need to obtain permission directly from the copyright holder. To view a copy of this licence, visit http://creativecommons.org/licenses/by/4.0/. The Creative Commons Public Domain Dedication waiver (http://creativecommons.org/publicdomain/zero/1.0/) applies to the data made available in this article, unless otherwise stated in a credit line to the data. 


\section{Background}

Dysmenorrhea (i.e., painful menstruation) is one of the most common gynecological problems and is characterized by acute pelvic pain during menstruation $[1,2]$. Depending on the pathological and anatomical statuses, dysmenorrhea is divided into two types of primary and secondary dysmenorrhea [3]. Primary dysmenorrhea is the presence of painful menstruation in the absence of pelvic diseases (e.g., endometriosis and pelvic inflammatory disease) or pelvic leiomyoma [4]. The results of a review of 15 primary studies published between 2002 and 2011 showed that the prevalence of primary dysmenorrhea ranged between 16 and 91\% in women over the age of 13, and severe dysmenorrhea was reported as being between 2 and 29\% [5]. According to a systematic review of Iranian studies (2016), the prevalence of primary dysmenorrhea was $71 \%$ [6]. Momenzadeh et al. reported that the prevalence of dysmenorrhea was $52.1 \%$ in Iranian girls under 15 [7].

Primary dysmenorrhea is the most common form of chronic pelvic pain that can last for at least 6 months, can be severe enough to disrupt one's daily life activities, and ultimately lead to medical or surgical treatment. Chronic pelvic pain associated with the patient's menstrual cycle is referred to as cyclic chronic pelvic pain [3]. Similar to other chronic pains, menstrual pain can act as a stressor and exacerbate psychological distress (e.g., the symptoms of depression and anxiety) [2].

During the management of dysmenorrhea, the relationship between dysmenorrhea and psychological problems are considered by most clinicians [8, 9]. The physical and psychological symptoms of primary dysmenorrhea (i.e., menstrual distress) may occur before, after, and during menstruation [10]. Menstrual distress is one of the most common problems in adolescence, with [11] 75 to $94 \%$ of women experiencing the symptoms [12]. Regarding treatment, 58-90\% of adult women prefer bed rest as a preventive treatment when they experience menstrual distress [13]. Menstrual distress is generally associated with symptoms such as irritability, tenderness of the breasts, low back pain, skin disorders, fatigue, palpitations, loneliness, nausea, vomiting, abdominal pain, and general weakness [14]. Painful menstruation and menstrual distress are associated with a deterioration of quality of life, as well as negative socioeconomic consequences [7, 15]. About $1 \%$ of women over the age of 18 are absent from work 1 to 3 days a month, due to severe dysmenorrhea. About $14 \%$ of adolescent girls miss school each month, due to painful menstruation [16, 17]. Given the impact on healthcare, the reduction in school attendance, and potential socioeconomic impacts of menstruation, this is an area that requires more recognition [5].
Despite various treatments for relieving menstrual pain and its associated symptoms, there is a need to find nonpharmacological approaches without causing side effects to adolescents' general health [4]. Various studies have shown the efficacy of complementary and alternative therapies (e.g., herbal remedies [18-20], exercise [11], heat [21, 22], and hypnosis [23]) at reducing menstrual pain. Cognitive behavioral therapy is one of the non-pharmacological treatments that can effectively relieve dysmenorrhea as psychological factors can exacerbate pain [24].

The Leventhal self-regulation model is used to understand illness perceptions [25] and provides a framework for the organization of individuals' perceptions about health, illness, and outcomes. This theory describes cognitive processes, emotional responses, and consequences of behaviors associated with health and illness [26]. Illness perceptions include responses to symptoms (e.g., weakness and lethargy) by directing cognitive and emotional experiences to related threats (e.g., dysmenorrhea) and guiding coping responses [27]. The model posits that people's cognitive perceptions about illness are related to five dimensions, which include beliefs about identity (e.g., related to diagnosis or symptoms of the disorder), causes (e.g., ideas about the origins), consequences (e.g., the impact on different aspects of life), timelines (e.g., thoughts about the duration of the disorder), and treatment or control (e.g., beliefs about the treatment and improvement of the disorder). Emotional perceptions also include negative reactions including fear, anxiety, anger, and discomfort [25].

Illness perceptions can affect health-related behaviors and adaptive behaviors that the patient requires to be able to manage, or cope with, a disease [28]. Research shows that a person's understanding of a disease is an important determinant of self-care [29]. A lack of awareness about one's illness can result in a type of vulnerability, which can in turn cause anxiety about the treatment process. In fact, education based on the self-regulation model can raise awareness, which can reduce anxiety and concerns [30].

The chronic nature of primary dysmenorrhea, its prevalence, and symptoms are major factors that disrupt quality of life and social activities for female adolescents. There is also a widely understood gender inequality in medical research resulting in a dearth of research on menstruation problems and women's health issues more generally [31]. Therefore, this study will aim to investigate the effects of psychoeducational intervention using the Leventhal self-regulation model during menstrual distress in adolescent girls with primary dysmenorrhea.

\section{Methods}

Study design and participants

The design of this randomized controlled trial is designed based on standards devised by the Consolidated 
Standards of Reporting Trials (CONSORT) (Fig. 1). Adolescent girls aged 14-19, attending high schools in Qazvin City, and experiencing moderate to severe pain (pain intensity $\geq 4$ based on the participant's VAS score for two consecutive months in a preliminary screening) will be recruited to the study. Inclusion criteria will include having regular menstruation and experiencing menstruation for at least 2 years. Girls will be excluded from the study if they have experienced secondary dysmenorrhea due to endometriosis, adenomyosis, subacute endometritis, pelvic inflammatory disease, intrauterine devices, ovarian cysts; congenital anomalies of the pelvis and stenosis; history of gynecological surgery; or report a history of mental or physical illnesses or substance abuse (based on their self-report and student's health reports). Participants will also be excluded if they are married or have taken specific medications for premenstrual syndrome (e.g., fluoxetine) in the past 6 months.

\section{Sample size estimation}

The estimation of the sample size is based on the study of Wong et al. considering the mean menstrual distress score for intervention group $23.96 \pm 4.79$ and $26.61 \pm 5.1$ in the control group [32], alpha $=0.05$, and $\beta=0.2$; the estimated sample size in each group will be 51 individuals in each group. Peng et al. suggested adding a 20\% attrition rate for youth, school-based studies [33], so the estimated sample size for the current study will be 60 individuals for each group. As we have two groups, the total sample will be 120 female students.

\section{Sampling and randomization}

Given the likelihood of information sharing between the students at the same school, the intervention and control groups will be selected from separate schools. A twostage cluster sampling design will be used to selected study samples. At the first stage, twelve schools will be

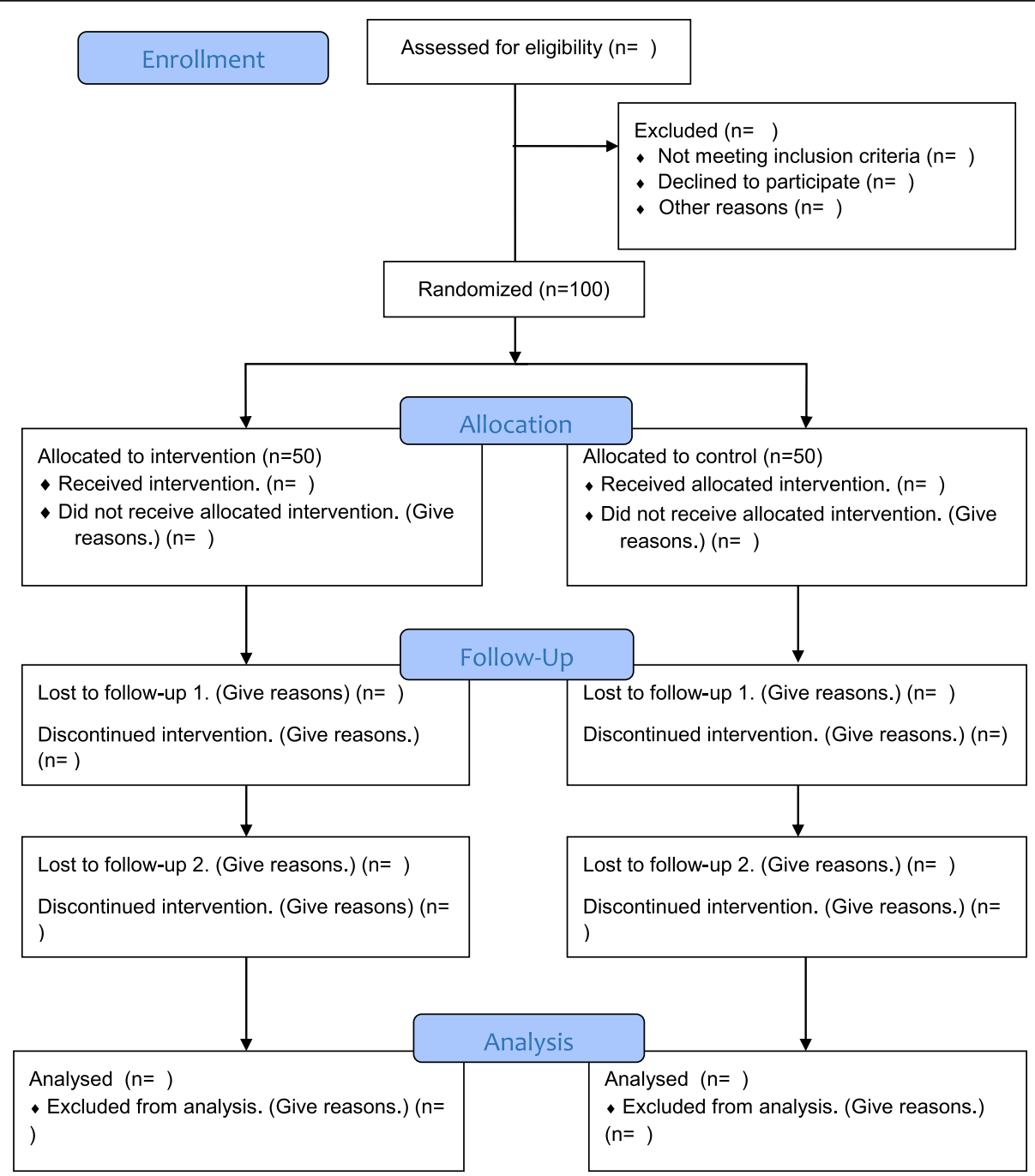

Fig. 1 CONSORT flow diagram 
randomly selected from a list of eligible schools (girlsspecific schools and not involved in similar educational intervention) in Qazvin city using a computer-generated random number. The list of schools will be provided by the department for Education in Qazvin. At the second stage, one class will be randomly selected from each school through a simple random method. Ten eligible individuals will be selected using random sampling method. Assigning of schools to the control and intervention groups will be done via random sampling method. Therefore, six schools will be assigned to the intervention, and six schools will be assigned to the control group.

\section{Intervention}

\section{Intervention sessions}

The sessions will be between 60 and 90 min apiece, and they will run for three consecutive weeks (one session per week). They will be held in groups consisting of 810 people [34]. In general, 5 intervention groups will be formed, and 15 educational sessions will be held at the school within 2 months of the sessions. If needed, individual counseling sessions on menstrual distress and common problems for adolescents will also be held.

\section{Intervention details}

The design of the intervention will be based on the Leventhal self-regulation model [30]. This model seeks to improve the perception of disease by increasing adolescents' awareness of their menstruation distress, as well as the control of their emotional responses and coping behavior. For this purpose, hypotheses about the selfregulation model will be used to design the training program. A self-regulated paradigm is a conscious personal management system that involves the process of guiding one's thoughts, behaviors, and emotions to achieve goals. Therefore, the educational material used for adolescent girls will be designed to be performed in three sessions:

The first session will be based on five dimensions of illness perception [35]. This stage is broken into four steps. The first step involves assessing the adolescent's perception of the nature of menstrual distress. This assessment includes psychological symptoms related to menstrual distress (e.g., anxiety and depression) and physical symptoms (e.g., fatigue, pain, and weakness). It also examines the beliefs of adolescents about the prevalence of menstrual distress and its complications (e.g., beliefs related to the identity of the disease). The second step will involve assessing the girls' perceptions about the cause of the menstrual distress and their beliefs about menstrual distress, in order to eliminate their untrue beliefs about the causes of this disease. Then, the duration of the illness and the adolescent's perception of the duration of the illness will be examined (i.e., the timeline). The third step will be concerned with any distress menstruation has on the adolescent's life (i.e., the consequences). During the last step, the researchers will examine the adolescent's perception of the efficacy of existing treatments, in order to control and improve the severity of menstrual pain and relieve symptoms of menstrual distress (e.g., ideas about treatment and improvement). This session will include techniques for selfcontrol, verbal encouragement, feedback, behavioral assessment, and ways to successfully control their illness experiences.

After reviewing the first session, the second session will begin. The adolescents will be asked to talk about their feelings and ambiguities about menstrual distress, and their beliefs and knowledge challenged by the researcher. The researchers will provide information to participants relating to how they could change their misperceptions about the disease. They will be asked if they had any questions about the previous session. In the last 30 min of each session, stress and relaxation strategies will be taught, and the adolescents will be asked to practice these techniques.

The third session will involve an evaluation and closure of the sessions. Beforehand, the adolescents will be notified that this session would be the last one. After they arrive, the content of the previous sessions will be reviewed. Then, they will be asked about the impact of new training and experiences on their lives. Problems and obstacles for each adolescent will be explored. Exercises from the previous sessions will be repeated, and their questions answered. Different methods will be used to increase the effectiveness of the training program.

To ensure adolescents are provided sufficient information to relate to the cognitive and psychological dimensions, a booklet will be used, in addition to face-to-face instructional education. The booklet will aim to improve illness perceptions by providing simple illustrations with explanations. The educational content of this booklet will identify and define menstrual distress, provide a brief explanation of misconceptions about the menstrual cycle, and offer activities authorized and unauthorized during the course and instructions about personal hygiene, physical activity, exercise, nutrition, stress-control techniques, and relaxation. The qualitative content of the booklet will be reviewed by ten faculty members. At the end of the three sessions, this booklet will be given to the intervention group. In addition, if participants had any further questions about the study or illness, the researcher's contact information will be provided to the adolescents.

\section{Data collection tools}

The following four tools will be used to collect data in the present study: 
1. Demographic and menstrual questionnaire: This tool will collect data about demographics related to menstrual problems, including age, age of menarche, education level, field of study, marital status, characteristics of the menstrual cycle (e.g., menstrual period and duration of bleeding), pain medication history, drug addiction, height, weight, and body mass index. It will also collect data about the adolescents' histories of dysmenorrhea or menstrual distress (e.g., in the mother or sister of the adolescent), secondary dysmenorrhea, underlying diseases, and vaginal sex. This questionnaire will be developed based on the aims of the study, and its validity will be evaluated using the content-validity method by the faculty members of midwifery at Qazvin University of Medical Sciences.

2. Visual analog scale (VAS): This valid, reliable tool will be used to assess the severity of dysmenorrhea [36]. The tool is a $100-\mathrm{mm}$ ruler, with zero at one end (which indicates complete pain relief) and ten on the other end (which indicated the most severe pain imaginable). Participants will mark the severity of pain on the ruler. This scale is commonly used in pain assessment, due to its ease of use [37].

3. Moos Menstrual Distress Questionnaire: This tool was designed by Radolf Moos [38] to investigate the impact of menstrual distress on one's daily activities. Its internal correlation is reported to be $83 \%$ for pain and $94 \%$ for autonomic activity. It consists of 47 questions, which are divided into 8 subgroups. It measures the patient's general complaints about various types of symptoms during menstruation. Participants will be asked to report symptoms experienced during menstruation, according to the Likert scoring system. A score of 1 for a condition will indicate no experience of any symptom, and 4 will suggest the most severe experience (i.e., almost debilitating). The short form of this questionnaire consisted of 16 to 19 questions. It was widely used to examine physical and psychological symptoms during the premenstrual, intra-menstrual, and intermenstrual phases in various contexts [22]. The validity and reliability of the Farsi version of this questionnaire were evaluated by Qorbanalipour et al. [39]. Therefore, it can be used in various studies for therapeutic purposes in the future.

4. Illness Perceptions Questionnaire: The IPQ was first developed by Weinman et al., based on the Leventhal model [40]. Its brief, 9-item, version will be used to assess emotional and cognitive perceptions about the disease which was modified to menstrual distress in this study. The first five questions examine the cognitive embodiment of the disease, including outcome, duration, individual control, treatment control, and nature. Two of the questions relate to affective visualization, including anxiety and emotional response. Scores range from 0 to 10, with higher scores indicating more severe misperceptions about the disease. The validity and reliability of the Farsi version of this questionnaire was confirmed by Bazazian and Beheshti.

\section{Primary outcome}

The primary outcome is the perceived menstrual distress of adolescent girls.

\section{Secondary outcomes}

The secondary outcomes are the illness perceptions and severity of dysmenorrhea pain.

\section{Procedure}

After obtaining the necessary permission to conduct the study, the researcher will attend the selected schools to identify eligible subjects and invite them to participate. The researchers will provide necessary explanations about the purpose of the research and ensure that data remains confidential. Informed consent will be obtained from eligible subjects. It will be explained that participants can withdraw from the research whenever they chose to. For two consecutive months prior to the intervention, an initial assessment of the severity of dysmenorrhea pain and menstrual distress will be performed in the groups. Questionnaires will be completed by the groups within 3 months after the intervention. Data will be collected using paper file storage. No intervention in the control group will be performed, but the educational content after the end of the study will be made available to the control group participants. If needed, the participants in both groups will be able to contact the researcher at any time. A small gift (a set of six colored pens) will be provided to participants to thank them for their participation. Figure 2 shows the timing of outcome measurement and intervention based on the SPIRIT table.

\section{Intervention fidelity}

The researcher (SA) is responsible for conducting the intervention and holding education sessions. At the time of the study, she is a midwife (BSc) and postgraduate student of counseling in midwifery. Therefore, she has sufficient experience in the field of research, and she has the necessary knowledge to conduct education and consulting sessions. Also, the educational sessions will be randomly evaluated by group instructors (NB and MAS) in $20 \%$ of the sessions. Specifically, initial meetings will 


\begin{tabular}{|c|c|c|c|c|c|c|c|c|}
\hline \multirow[b]{3}{*}{ TIMEPOINT } & \multicolumn{8}{|c|}{ STUDY PERIOD } \\
\hline & \multirow{2}{*}{ Enrolment } & \multirow{2}{*}{ Allocation } & \multicolumn{3}{|c|}{$\begin{array}{c}\text { Post-allocation (Intervention } \\
\text { period }^{*} \text { ) }\end{array}$} & \multicolumn{3}{|c|}{$\begin{array}{c}\text { Fallow Up after } \\
\text { intervention completed }\end{array}$} \\
\hline & & & $\begin{array}{c}\begin{array}{c}\text { First } \\
\text { session }\end{array} \\
\text { sess }\end{array}$ & $\begin{array}{l}\text { Second } \\
\text { session }\end{array}$ & $\begin{array}{l}\text { Third } \\
\text { session }\end{array}$ & $\begin{array}{c}1 \\
\text { month }\end{array}$ & $\begin{array}{c}2 \\
\text { month }\end{array}$ & $\begin{array}{c}3 \\
\text { month }\end{array}$ \\
\hline \multicolumn{9}{|l|}{ ENROLMENT: } \\
\hline Eligibility screen & $\mathrm{x}$ & & & & & & & \\
\hline Informed consent & $x$ & & & & & & & \\
\hline $\begin{array}{l}\text { Randomized by } \\
\text { independent person }\end{array}$ & $\mathrm{x}$ & & & & & & & \\
\hline Allocation & & $x$ & & & & & & \\
\hline \\
\hline \multirow{2}{*}{\multicolumn{9}{|c|}{$\begin{array}{l}\text { Group A: } \\
\text { Psychoeducational } \\
\text { Intervention } \\
\text { Group B: Control }\end{array}$}} \\
\hline & & & & & & & & \\
\hline \multicolumn{9}{|l|}{ ASSESSMENTS: } \\
\hline $\begin{array}{l}\text { Dysmenorrhea } \\
\text { severity }\end{array}$ & $\mathrm{x}$ & & & & & $x$ & $\mathrm{X}$ & $\mathrm{X}$ \\
\hline Menstrual distress & $x$ & & & & & $x$ & $x$ & $x$ \\
\hline
\end{tabular}

Fig. 2 SPIRIT schedule of enrolment, interventions, and follow-up assessments

be held to ensure the researcher's ability to execute the educational sessions.

\section{Data management}

The researcher (SA) will be responsible for monitoring the data collection process throughout the study process. Data entry into the SPSS software will be controlled by ZA and NB.

\section{Data analysis method}

Collected data will be analyzed on an intention-to-treat basis using MLwin 2.27 [41]. ICC will be calculated within groups and a three-level mixed model will be used with adolescents girls nested in schools. The significance level will be $p<0.05$. Finally, power of study will be calculated too.

\section{Discussion}

This study will be the first randomized controlled trial to treat menstrual distress in adolescent girls that utilizes a psychoeducational intervention based on the Leventhal self-regulation model. The study results could provide insights into the effectiveness of using this model to treat menstrual distress in adolescents. Other interventions have focused on the effectiveness of reducing the severity of menstrual cramps using yoga [42], hypnosis [43], and the education of self-care behavior [44], for example. However, few other studies have been conducted on this topic, and no interventions have been performed to reduce menstrual distress, in addition to reducing the severity of menstrual pain. Given the high prevalence and impact of the physical and psychological complications of menstrual distress on adolescent academic performance, more interventions are still needed. Fortunately, education based on the Leventhal model of 
self-regulation is uncomplicated, low-cost, and easy to use. This model has also been used to reduce psychological distress, perceived stress, and anxiety in individuals with chronic diseases, and it has shown beneficial effects $[45,46]$. If this intervention shows promising effects, it can reduce the rate of school abstinence and increase student's academic achievements. More specifically, this study will be designed to focus on the chronic nature of dysmenorrhea and shape the specific understanding of how to manage menstrual distress by using the self-regulation model.

\section{Abbreviations}

CONSORT: Consolidated Standards of Reporting Trials; IPQ: IIness Perceptions Questionnaire; VAS: Visual analog scale

\section{Acknowledgements}

Vice chancellor (Research) of Qazvin University of Medical Sciences has provided financial support to this project.

\section{Trial status}

The recruitment has not yet begun; however, all necessary permissions have been acquired; the estimated date of recruitment is December 30, 2019. The expected time for completing the recruitment is July 15, 2020. Protocol version 1 registered 2019-09-03.

\section{Authors' contributions}

All authors contributed to the conception and design of the study. SA and NB drafted the manuscript. ZA, MAS, and KAA provided contributions to the literature review and substantially edited the primary manuscript and prepared the final version of the manuscript. All authors revised the manuscript, agreed to be fully accountable for ensuring the integrity and accuracy of the study, and read and approved the final version of the manuscript to be published. All the authors met the criteria for authorship, and they are listed as co-authors on the title page.

\section{Funding}

The Qazvin University of Medical Sciences financially supports the project, but the funding body does not have any role in the design, data collection, data analysis, and interpretation of findings.

\section{Availability of data and materials}

After performing the main study, the analyzed data and materials will be deidentified and published.

\section{Ethics approval and consent to participate}

The research protocol is approved by the Research Review Board at Qazvin Faculty of Nursing and Midwifery (IR.QUMS.REC. 1398.043 at the Human Ethics Committee at Qazvin University of Medical Sciences). The permissions to access the health and medical centers have been obtained from the authorities in Qazvin University of Medical Sciences. The researcher will introduce herself to the participants. After introducing the research objectives, the confidentiality of data maintenance, and the freedom to withdraw from the study, the written informed consent form will be signed by the participants who are willing to participate in this study. Because the age of adolescents in the study is under 18 years, in addition to obtaining informed consent from the adolescent, the purpose, method of study, and potential advantages and disadvantages of participating in the study will also be explained to their parents. Then, the informed consent will be obtained from the parents.

\section{Consent for publication}

Not applicable

\section{Competing interests}

None to declare

\section{Author details}

'Student Research Committee, Qazvin University of Medical Sciences, Qazvin, Iran. ${ }^{2}$ Social Determinants of Health Research Center, Research Institute for Prevention of Non-Communicable Diseases, Qazvin University of Medical Sciences, Shahid Bahonar Blvd, Qazvin 3419759811, Iran. ${ }^{3}$ Educational Psychology and Inclusive Education, Faculty of Education, Monash University and The Centre for Positive Psychology, The Melbourne Graduate School of Education, The University of Melbourne, Melbourne, Australia.

Received: 6 November 2019 Accepted: 26 July 2020

Published online: 27 August 2020

\section{References}

1. Bair MJ, Robinson RL, Katon W, Kroenke K. Depression and pain comorbidity: a literature review. Arch Intern Med. 2003;163(20):2433-45.

2. Rapkin A. Pelvic pain and dysmenorrhea. Berek Novaks Gynecol. 2012;475: 470-4.

3. Jonathan SB, Berek B, Paoulo AB, editors. Novak's gynecology. Maryland, library of congress cataloging; 2012.

4. lacovides SAI, Baker FC. What we know about primary dysmenorrhea today: a critical review. Hum Reprod Update. 2015;21(6):762-78.

5. Ju H, Jones M, Mishra G. The prevalence and risk factors of dysmenorrhea. Epidemiol Rev. 2014;36(1):104-13.

6. Kharaghani R, Damghanian MJIRCMJ. The prevalence of dysmenorrhea in iran: a systematic review and meta-analysis. 2017;19(3):1-10.

7. Momenzadeh FTM, Taghizadeh M, Mahlioji M, Rafiee F. Comparison the effect of fennel and mefenamic acid on severity of primary dysmenorrhea. Iran J Obstet Gynecol Infertility. 2017;20(4):44-9.

8. Sahin N, Kasap B, Kirli U, Yeniceri N, Topal Y. Assessment of anxietydepression levels and perceptions of quality of life in adolescents with dysmenorrhea. Reprod Health. 2018;15(1):13.

9. Bajalan Z, Moafi F, MoradiBaglooei M, Alimoradi Z. Mental health and primary dysmenorrhea: a systematic review. J Psychosom Obstet Gynecol. 2019;40(3):185-94

10. Omidvar S, Begum K. Menstrual pattern among unmarried women from south India. J Nat Sci Biol Med. 2011;2(2):174.

11. Shahr-Jerdy SHR, Eivazi M. Effects of stretching exercises on primary dysmenorrhea in adolescent girls. Biomed Hum Kinetics. 2012;4:32-127.

12. Chen $\mathrm{H}-\mathrm{M}$, Wang $\mathrm{H}-\mathrm{H}$, Chiu $\mathrm{M}-\mathrm{H}, \mathrm{Hu} \mathrm{H}-\mathrm{M}$. Effects of acupressure on menstrual distress and low back pain in dysmenorrheic young adult women: an experimental study. Pain Manag Nurs. 2015;16(3):188-97.

13. Al-Kindi R, Al-Bulushi A. Prevalence and impact of dysmenorrhoea among Omani high school students. Sultan Qaboos Univ Med J. 2011;11(4):485.

14. Kordi M, Mohamadirizi S, Shakeri MT, Salehi Fadardi J, Hafizi L. The relationship between midwives' work stress and perimenstrual distress. Iran J Obstet Gynecol Infertility. 2011;14(3):54-63.

15. Gharloghi S, Torkzahrani S, Akbarzadeh AR, Heshmat R. The effects of acupressure on severity of primary dysmenorrhea. Patient Preference Adherence. 2012;6:137

16. Gagua TTB, Gagua D, McHedlishvili N. Assessment of anxiety and depression in adolescents with primary dysmenorrhea:a case-control study. J Pediatr Adolesc Gynecol. 2013;26(6):350-4.

17. Haidari FAA, Sarhadi M, Mohammad SM. Prevalence and severity of primary dysmenorrhea and its relation to anthropometric parameters. J Hayat. 2011; 17(1):70-7.

18. Davdabady Farahani MK, Seyyedzadeh Aghdam N. Comparison of ginger and valerian on the severity of primary dysmenorrhea: a randomized triple blind clinical trial. Complement Med J Fac Nurs Midwifery. 2013;3(2):494503 [Persian].

19. Kazaeeian SNA, Navabi RS. Evaluation effects of oral aloe vera gel on the intensity of primery dysmenorrhea. Med Surg Nurs Q. 2012;1(1):35-40.

20. Rasoolzadeh NZJ, Zolphagari M, Mehran A. Effects of relaxation on primary dysmenorhea among first year nursing and midwifery female students. Hayat. 2007;13(2):2330

21. Hosono TTY, Morita $Y$, Nishimura $Y$, Sugita $Y$, Isami C. Effects of a heat-and steam-generating sheet on relieving symptoms of primary dysmenorrhea in young women. J Obstet Gynaecol Res. 2010;36(4):24-818.

22. DA O'Connell K, Westhoff C. Self-treatment patterns among adolescent girls with dysmenorrhea. J Pediatr Adolesc Gynecol. 2006;19(4):285-9.

23. Farshbaf Manei Sefat F, Abolghasemi A, Barahmand U, Hajloo N. Comparing the effectiveness of cognitive behavioral therapy and hypnosis therapy pain 
self-efficacy and pain severity in girls with primary dysmenorrhea. J Armaghane Danesh. 2017;22(1):87-103.

24. Vlaeyen JWCG, Goubert L. The psychology of chronic pain and its management. Phys Ther Rev. 2007;12(3):179-88.

25. McSharry J, Moss-Morris R, Kendrick T. Illness perceptions and glycaemic control in diabetes: a systematic review with meta-analysis. Diabet Med. 2011;28(11):1300-10.

26. Rakhshan M. In: Rakhshan ME, editor. The application of the Lorentz selfgoverning theory in nursing. Mashhad: Young Nurses; 2014.

27. Broadbent E, Ellis CJ, Thomas J, Gamble G, Petrie KJ. Further development of an illness perception intervention for myocardial infarction patients: a randomized controlled trial. J Psychosom Res. 2009;67(1):17-23.

28. Rahimi Z, Baljani S, Zadgasem Z. Investigating the relationship between illness perception and quality of life in hemodialysis patients; 2012.

29. Abubakari AR, Jones MC, Lauder W, Kirk A, Anderson J, Devendra D. Associations between knowledge, illness perceptions, self-management and metabolic control of type 2 diabetes among African and European-origin patients. J Nurs Healthc Chronic IIIn. 2011;3(3):245-56.

30. Cameron LD, Leventhal $H$. The self-regulation of health and illness behaviour. Canada and USA: Psychology Press; 2003.

31. Holdcroft A. Gender bias in research: how does it affect evidence based medicine? London: SAGE Publications Sage UK; 2007.

32. Wong CL, Lai KY, Tse HM. Effects of SP6 acupressure on pain and menstrual distress in young women with dysmenorrhea. Complement Ther Clin Pract. 2010;16(2):64-9.

33. Peng C-YJ, Harwell M, Liou S-M, Ehman LH. Advances in missing data methods and implications for educational research. Real Data Anal. 2006;3178.

34. Zakerimoghadam M, Sadeghi-Akbari A, Cheraghi M-A, Kazemnejad A. The effect of education based on illness perception on self-care behaviors of patients with heart failure. IJCN. 2015;4(1):22-30.

35. Kus T, Aktas G, Ekici H, Elboga G, Djamgoz S. Illness perception is a strong parameter on anxiety and depression scores in early-stage breast cancer survivors: a single-center cross-sectional study of Turkish patients. Support Care Cancer. 2017;25(11):3347-55

36. Zahedifard TFM. Assessment of the articles related to primary dysmenorrhea in dimension of menstrual pain assessment tools. Iran J Obstet Gynecol Infertility. 2016:19(4):17-27.

37. Mudgalkar N, Bele SD, Valsangkar S, Bodhare TN, Gorre MJI. Utility of numerical and visual analog scales for evaluating the post-operative pain in rural patients. Indian J Anaesth. 2012;56(6):553.

38. Moos RH. The development of a menstrual distress questionnaire. Psychosom Med. 1968;30(6):853-67.

39. Qorbanalipour K, Ghaderi F, Asghari Jafarabadi M, Mohammadalizade Charandabi S. Validity and reliability of the Persian version of modified Moos Menstrual Distress Questionnaire \%J The Iranian Journal of Obstetrics, Gynecol Infertility 2016;19(29):11-18.

40. Valipoor F, Rezaei F. Assessment of Illness perception in the patients with diabetes mellitus and its association with control of blood sugar in the patients referring to Tohid Hospital in Sanandaj city. HBI J. 2013;18(2):9-17.

41. Rasbash J, Steele F, Browne W, Goldstein H, Charlton C, Browne W. A user's quide to MLwiN, V2. 26. centre for multilevel modelling. UK: University of Bristol; 2012.

42. Yang N-Y, Kim S-DJTJA, Medicine C. Effects of a yoga program on menstrual cramps and menstrual distress in undergraduate students with primary dysmenorrhea: a single-blind, randomized controlled trial. 2016;22(9):732-8.

43. Bazzazian S, Besharat MA. Reliability and validity of a Farsi version of the brief illness perception questionnaire. Procedia Soc Behav Sci. 2010;5:962-5.

44. Kabirian A, Abedian Z, Mazloum SR, Kabirian MJJTHUMS. The effect of selfcare behaviors based on evidence-based education on the severity of dysmenorrhea amongst girls. 2017;5(1):26-31.

45. Morgan K, Villiers-Tuthill A, Barker M, McGee H. The contribution of illness perception to psychological distress in heart failure patients. BMC Psychol. 2014;2(1):50.

46. Timmermans I, Versteeg H, Meine M, Pedersen S, Denollet J. Illness perceptions in patients with heart failure and an implantable cardioverter defibrillator: dimensional structure, validity, and correlates of the brief illness perception questionnaire in Dutch, French and German patients. J Psychosom Res. 2017;97:1-8.

\section{Publisher's Note}

Springer Nature remains neutral with regard to jurisdictional claims in published maps and institutional affiliations.

\section{Ready to submit your research? Choose BMC and benefit from}

- fast, convenient online submission

- thorough peer review by experienced researchers in your field

- rapid publication on acceptance

- support for research data, including large and complex data types

- gold Open Access which fosters wider collaboration and increased citations

- maximum visibility for your research: over $100 \mathrm{M}$ website views per year

At BMC, research is always in progress.

Learn more biomedcentral.com/submissions 